\title{
GRNsight: a web application and service for visualizing models of small- to medium-scale gene regulatory networks
}

\author{
Kam D Dahlquist ${ }^{\text {Corresp.., }}{ }^{1}$, John David N Dionisio ${ }^{2}$, Ben G Fitzpatrick ${ }^{3}$, Nicole A Anguiano ${ }^{2}$, Anindita \\ Varshneya $^{1}$, Britain J Southwick ${ }^{2}$, Mihir Samdarshi ${ }^{1}$ \\ 1 Department of Biology, Loyola Marymount University, Los Angeles, California, United States \\ 2 Department of Electrical Engineering and Computer Science, Loyola Marymount University, Los Angeles, California, United States \\ 3 Department of Mathematics, Loyola Marymount University, Los Angeles, California, United States \\ Corresponding Author: Kam D Dahlquist \\ Email address: kdahlquist@Imu.edu
}

GRNsight is a web application and service for visualizing models of gene regulatory networks (GRNs). A gene regulatory network consists of genes, transcription factors, and the regulatory connections between them which govern the level of expression of mRNA and protein from genes. The original motivation came from our efforts to perform parameter estimation and forward simulation of the dynamics of a differential equations model of a small GRN with 21 nodes and 31 edges. We wanted a quick and easy way to visualize the weight parameters from the model which represent the direction and magnitude of the influence of a transcription factor on its target gene, so we created GRNsight. GRNsight automatically lays out either an unweighted or weighted network graph based on an Excel spreadsheet containing an adjacency matrix where regulators are named in the columns and target genes in the rows, a Simple Interaction Format (SIF) text file, or a GraphML XML file. When a user uploads an input file specifying an unweighted network, GRNsight automatically lays out the graph using black lines and pointed arrowheads. For a weighted network, GRNsight uses pointed and blunt arrowheads, and colors the edges and adjusts their thicknesses based on the sign (positive for activation or negative for repression) and magnitude of the weight parameter. GRNsight is written in JavaScript, with diagrams facilitated by D3.js, a data visualization library. Node.js and the Express framework handle server-side functions. GRNsight's diagrams are based on D3.js's force graph layout algorithm, which was then extensively customized to support the specific needs of GRNs. Nodes are rectangular and support gene labels of up to 12 characters. The edges are arcs, which become straight lines when the nodes are close together. Self-regulatory edges are indicated by a loop. When a user mouses over an edge, the numerical value of the weight parameter is displayed. Visualizations can be modified by sliders that adjust the force graph layout parameters and through manual node dragging. GRNsight is best-suited for visualizing networks of fewer than 35 nodes and 70 
edges, although it accepts networks of up to 75 nodes or 150 edges. GRNsight has general applicability for displaying any small, unweighted or weighted network with directed edges for systems biology or other application domains. GRNsight serves as an example of following and teaching best practices for scientific computing and complying with FAIR Principles, using an open and test-driven development model with rigorous documentation of requirements and issues on GitHub. An exhaustive unit testing framework using Mocha and the Chai assertion library consists of around 160 automated unit tests that examine nearly 530 test files to ensure that the program is running as expected. The GRNsight application (http://dondi.github.io/GRNsight/) and code (https://github.com/dondi/GRNsight) are available under the open source BSD license. 
1 GRNsight: a web application and service for visualizing models of

2 small- to medium-scale gene regulatory networks

3 Kam D. Dahlquist ${ }^{1 *}$, John David N. Dionisio ${ }^{2}$, Ben G. Fitzpatrick ${ }^{3}$, Nicole A. Anguiano ${ }^{2}$,

4 Anindita Varshneya ${ }^{1}$, Britain J. Southwick ${ }^{2}$, Mihir Samdarshi ${ }^{1}$

$5 \quad{ }^{1}$ Department of Biology, ${ }^{2}$ Department of Electrical Engineering and Computer Science,

$6 \quad{ }^{3}$ Department of Mathematics, Loyola Marymount University, 1 LMU Drive, Los Angeles, CA

790045 USA

$8 \quad$ *Corresponding author:

9 Kam D. Dahlquist

10 Department of Biology

11 Loyola Marymount University

121 LMU Drive, MS8888

13 Los Angeles, CA 90045 USA

14 E-mail: kdahlquist@lmu.edu

15 Tel: 1-310-338-7697

16 Link to web application: http://dondi.github.io/GRNsight/

17 Link to code repository: https://github.com/dondi/GRNsight 
18 Abstract

GRNsight is a web application and service for visualizing models of gene regulatory networks (GRNs). A gene regulatory network consists of genes, transcription factors, and the regulatory connections between them which govern the level of expression of mRNA and protein from genes. The original motivation came from our efforts to perform parameter estimation and forward simulation of the dynamics of a differential equations model of a small GRN with 21 nodes and 31 edges. We wanted a quick and easy way to visualize the weight parameters from the model which represent the direction and magnitude of the influence of a transcription factor on its target gene, so we created GRNsight. GRNsight automatically lays out either an unweighted or weighted network graph based on an Excel spreadsheet containing an adjacency matrix where regulators are named in the columns and target genes in the rows, a Simple Interaction Format (SIF) text file, or a GraphML XML file. When a user uploads an input file specifying an unweighted network, GRNsight automatically lays out the graph using black lines and pointed arrowheads. For a weighted network, GRNsight uses pointed and blunt arrowheads, and colors the edges and adjusts their thicknesses based on the sign (positive for activation or negative for repression) and magnitude of the weight parameter. GRNsight is written in JavaScript, with diagrams facilitated by D3.js, a data visualization library. Node.js and the Express framework handle server-side functions. GRNsight's diagrams are based on D3.js's force graph layout algorithm, which was then extensively customized to support the specific needs of GRNs. Nodes are rectangular and support gene labels of up to 12 characters. The edges are arcs, which become straight lines when the nodes are close together. Self-regulatory edges are indicated by a loop. When a user mouses over an edge, the numerical value of the weight parameter is displayed. Visualizations can be modified by sliders that adjust the force graph 
41 layout parameters and through manual node dragging. GRNsight is best-suited for visualizing

42 networks of fewer than 35 nodes and 70 edges, although it accepts networks of up to 75 nodes or

$43 \quad 150$ edges. GRNsight has general applicability for displaying any small, unweighted or weighted

44 network with directed edges for systems biology or other application domains. GRNsight serves

45 as an example of following and teaching best practices for scientific computing and complying

46 with FAIR Principles, using an open and test-driven development model with rigorous

47 documentation of requirements and issues on GitHub. An exhaustive unit testing framework

48 using Mocha and the Chai assertion library consists of around 160 automated unit tests that

49 examine nearly 530 test files to ensure that the program is running as expected. The GRNsight

50 application (http://dondi.github.io/GRNsight/) and code (https://github.com/dondi/GRNsight) are

51 available under the open source BSD license. 


\section{Introduction}

GRNsight is a web application and service for visualizing models of small- to medium-

54 scale gene regulatory networks (GRNs). A gene regulatory network consists of genes,

transcription factors, and the regulatory connections between them which govern the level of expression of mRNA and protein from genes. Our group has developed a MATLAB program to perform parameter estimation and forward simulation of the dynamics of an ordinary differential equations model of a medium-scale GRN with 21 nodes and 31 edges (Dahlquist et al., 2015; http://kdahlquist.github.io/GRNmap/). GRNmap accepts a Microsoft Excel workbook as input, with multiple worksheets specifying the different types of data needed to run the model. For compactness, the GRN itself is specified by a worksheet that contains an adjacency matrix where regulators are named in the columns and target genes in the rows. Each cell in the matrix contains a " 0 " if there is no regulatory relationship between the regulator and target, or a " 1 " if there is a regulatory relationship between them. The GRNmap program then outputs the estimated weight parameters in a new worksheet containing an adjacency matrix where the " 1 's" are replaced with a real number that is the weight parameter, representing the direction (positive for activation or negative for repression) and magnitude of the influence of the transcription factor on its target gene (Dahlquist et al., 2015). Although MATLAB has graph layout capabilities, we wanted a way for novice and experienced biologists alike to quickly and easily view the unweighted and weighted network graphs corresponding to the matrix without having to create or modify MATLAB code. Given that our user base included students in courses using university computer labs where the installation and maintenance of software is subject to logistical considerations sometimes beyond our control, we enumerated the following requirements for a potential visualization tool. The tool should: 
1. Exist as a web application without the need to download and install specialized software;

2. Be simple and intuitive to use;

3. Accept an input file in Microsoft Excel format (.xlsx);

4. Read a weighted or unweighted adjacency matrix where the regulatory transcription factors are in columns and the target genes are in rows;

5. Automatically lay out and display small- to medium-scale, unweighted and weighted, directed network graphs in a way that is familiar to biologists and adds value to the interpretation of the modeling results.

Having established the broad technical requirements to which we were seeking a solution, the first task was to determine if software already existed that could fulfill our needs. A review by Pavlopoulos et al., published in 2015, describes the types, trends, and usage of visualization tools available for genomics and systems biology. Their list of 47 tools for network analysis is representative of what was available to us at our project inception in January 2014 (given the caveat that the list itself is a moving target with some tools dropping out, new ones being added, and others evolving in their functions). With such a large number of tools available, it would be reasonable to expect that one already existed that could fulfill our needs. However, our use case was narrow, and the tools we investigated out of this diverse set each had properties that limited their use for us. With regard to our first requirement, out of the 47 tools, 29 are stand-alone applications, requiring installation, versus 18 web applications. With respect to our second requirement, the more complex software packages out of the set have a steep learning curve. Our third and fourth requirements specify data types. Some packages were hardcoded for a different type of network than a GRN (e.g., metabolic or signaling pathways, protein-protein interaction networks) or retrieved data exclusively from a backend database, not 
98 allowing user-supplied data. None at the time would readily accept an adjacency matrix with the

99 GRNmap specifications as input without some manipulation of the data format. Finally, with

100 respect to the last requirement, the core functionality, some packages were designed for

101 visualization and analysis of much larger networks than the ones in which we were interested or

102 did not have the ability to display directed, weighted graphs.

As an illustration of this, Pavlopoulos et al. (2015) showed that the open source software, Cytoscape (Shannon et al., 2003; Smoot et al., 2011) had the highest citation count in the Scopus database, as it is widely recognized as the "best-in-class" tool for viewing and analyzing large networks for systems biology research. However, while Cytoscape is flexible in terms of what types of network representations it accepts as input (SIF, NNF, GML, XGMML, SBML, BioPAX, PSI-MI, GraphML, cf. http://manual.cytoscape.org/en/latest/Supported_Network_File_Formats.html\#supportednetwork-file-formats), its basic "unformatted table files" format expects the network to be

111 represented in a list of pairwise interactions between two nodes instead of as an adjacency matrix, requiring a GRNmap user to convert the file external to the program. Furthermore,

113 Cytoscape must be installed on a user's computer. Finally, because it is powerful and has a lot of 114 features, there is a somewhat steep learning curve before a novice user can begin to visualize networks. Multiple settings must be learned and selected to generate a display that properly fits a use case; it is not possible to just "load into Cytoscape and go." Another open source application, Gephi (Bastian, Heymann, and Jacomy, 2009), is a general graph visualization tool that does accept an adjacency matrix in .csv format (among a wide range of supported formats, cf. https://gephi.org/users/supported-graph-formats/csv-format/), but again requires download and installation of the software and has a complex feature set. Because GRNmap itself is 
121 complex software targeted both at experienced biology investigators and novice undergraduate

122 users in a Biomathematical Modeling course, we wanted to limit the need to install and learn

123 additional visualization software. Reducing the cognitive load required for using the software

124 would allow users to focus their attention on understanding the biological results of the model.

125 After this exploration, we decided to create our own software solution that we could

126 exactly tailor to our specifications. Following the philosophy of "do one thing well"

127 (http://onethingwell.org/post/457050307/about-one-thing-well), we wanted to prioritize

128 rendering small- to medium-scale gene regulatory networks both easily and well. It was more

129 important for us to create a tool that is specifically tailored to the visualization of these sized

130 GRNs, and not every possible graph from every possible application domain. Similarly, we

131 wanted to pass data seamlessly from GRNmap to GRNsight, while bearing in mind that we

132 should adopt practices that would also make our tool useful to users outside our own group.

133 Finally, we wanted to minimize any startup, onboarding, or overhead time, which necessitated

134 also enumerating a set of process requirements that would lead us to our goal. Our project

135 should:

136 - Follow best practices for open software development in bioinformatics, including:

137 reusing code, releasing early and often to a public repository, tracking requirements,

138 issues, and bugs, performing unit-tests, and providing both code and user documentation

139 (Schultheiss, 2011; Prlic and Procter, 2012; Wilson et al., 2014);

140 - Leverage the expertise of the faculty and undergraduate student development team

141 members and be responsive to our GRNmap customers (i.e., eat our own dog food); 
142

143

144

145

146

147

148

150

151

152

- Balance the needs of fulfilling our own use case with developing a tool of wider applicability to the scientific community during a development cycle that ebbs and flows with the pressures of the academic calendar.

GRNsight both fulfills our stated product requirements and serves as a model for best practices for software development in bioinformatics as discussed in the sections below.

\section{Materials and Methods}

Input Data

GRNsight automatically lays out the network graph specified by an adjacency matrix contained within a worksheet named "network" or "network_optimized_weights" in a Microsoft Excel workbook (.xlsx). It was designed to accept workbooks seamlessly from the MATLAB gene regulatory network modeling program, GRNmap; however, the expected input format is general and is not dependent on GRNmap. Detailed documentation for the expected input file format is found on the GRNsight Documentation page:

http://dondi.github.io/GRNsight/documentation.html.

$$
\text { GRNsight can automatically lay out either an unweighted or weighted network graph }
$$
specified by an adjacency matrix where regulators are named in the columns and target genes in the rows. Note that regulators (regulatory transcription factors) are themselves encoded by genes and will be referred to as such. The adjacency matrix can be either symmetric (with the exact same genes named in both the columns and rows) or asymmetric (additional genes in either the columns or rows or both). For an unweighted network, each cell in the matrix should contain a " 0 " if there is no regulatory relationship between the regulator and target, or a " 1 " if there is a 
163 regulatory relationship between them (Fig. 1). In a weighted network, the "1's" are replaced

164 with a real number that is the weight parameter (Fig. 2). Positive weights indicate activation of

165 the target gene by the regulator, and negative weights indicate repression of the target gene by

166 the regulator.

After having implemented the core functionality of seamlessly reading GRNmapgenerated Excel workbooks, we recently extended the ability of GRNsight to read other commonly used network data formats to increase the interoperability of GRNsight with other network analysis and visualization software. GRNsight can import and display Simple

171 Interaction Format (SIF, .sif, http://manual.cytoscape.org/en/latest/Supported_Network_File_Formats.html\#sif-format) and GraphML (.graphml; Brandes et al., 2001; http://graphml.graphdrawing.org/) files and export network data in those two formats (see the GRNsight Documentation page for details of the implementation at http://dondi.github.io/GRNsight/documentation.html). regulatory network for an organism. The bounding box for display of the graph has a fixed size.

178 Currently, it is recommended that the user upload networks with no more than 35 unique genes 179 (nodes) or 70 edges. A warning is given upon upload of a network with 50-74 nodes or 71-99 180 edges, although the network graph will still display. If the user attempts to upload a network of 18175 or more nodes or 100 or more edges, the graph does not display, and an error message will be 182 returned. 
183

184

185

186

187

188

189

190

191

192

193

194

195

196

197

198

Architecture

GRNsight has a service-oriented architecture, consisting of separate server and web client components (Fig. 3). The server provides a web API (application programming interface) that accepts a Microsoft Excel workbook (.xlsx) file via a POST request and converts it into a corresponding JSON (JavaScript Object Notation) representation. Conversion is accomplished by first parsing the .xlsx file using the node-xlsx library (https://github.com/mgcrea/node-xlsx) then mapping the translated worksheet cells into JSON. It also provides demonstration graphs already in this JSON format, without requiring a spreadsheet upload. The web client provides a graphical user interface for visualizing the JSON graphs provided by the server, whether the graphs are parsed from uploaded Excel workbooks or provided directly by the server's demos. As an additional layer of customization, the graphical interface provided by the web client can be embedded in any web page using the standard iframe element. This is the mechanism used in deploying the production and beta versions of the software on https://dondi.github.io/GRNsight. Figure 3 illustrates this architecture and the interactions of the components. Documentation for how GRNsight is specifically deployed, including autonomous production and beta versions, can be found on the GRNsight wiki (https://github.com/dondi/GRNsight/wiki/Server-Setup).

GRNsight is an open source project and is itself built using other open source software. Server-side components are implemented with Node.js and the Express framework (Brown, 2014). Graph visualization is facilitated by the Data-Driven Documents JavaScript library (D3.js; Bostock, Ogievetsky, and Heer, 2011). D3.js provides data mapping and layout routines which GRNsight heavily customizes in order to achieve the desired graph visualization. The resulting graph is a Scalable Vector Graphics (SVG) drawing in which D3.js maps gene objects from the JSON representation provided by the web API server onto labeled rectangles. Edge 
206 weights are mapped into Bezier curves. The resulting graph is interactive, initially using D3.js's

207 force graph layout algorithm to automatically determine the positions of the gene rectangles.

208 The user can then drag the rectangles to improve the graph's layout. Customizations to the graph

209 display are described further in the next section.

As noted in the Introduction, we decided to create our own GRNsight software instead of

211 utilizing prior existing network visualization packages, like Cytoscape (Shannon et al., 2003;

212 Smoot et al., 2011). However, in keeping with open source development practices, we did

213 leverage other pre-existing code as described above. Besides D3.js, Cytoscape.js (Franz et al.,

214 2016) has been developed as an open source network visualization engine. The BioJS registry

215 (Yachdav et al., 2015) also lists a dozen components tagged with the keyword "network." The

216 choice of D3.js as the visualization engine was made simply to leverage the expertise of one of

217 the co-authors who was already familiar with the D3.js library in order to minimize the startup,

218 onboarding, and overhead time for the project, which initially served as a semester-long capstone

219 experience for one of the undergraduate co-authors.

\section{Graph Customizations}

GRNsight's diagrams are based on D3.js's force graph layout algorithm (Bostock,

Ogievetsky, and Heer, 2011), which was then extensively customized to support the specific needs of biologists for GRN visualization. D3.js's baseline force graph implementation had round, unlabeled nodes and undirected, straight-line edges. The following customizations were made for the nodes: (a) the nodes were made rectangular; (b) a label of up to 12 characters was added; (c) node size was varied, depending on the size of the label. 

segments, the edges display as directed edges. They are implemented as Bezier curves that straighten when nodes are close together and curve when nodes are far apart. A special case was added to form a looping edge if a node regulated itself. When an unweighted adjacency matrix is uploaded, all edges are displayed as black with pointed arrowheads. When a weighted adjacency matrix is uploaded, edges are further customized based on the sign and magnitude of the weight parameter. As is common practice in biological pathway diagrams (Gostner et al., 2014), activation (for positive weights) is represented by pointed arrowheads, and repression (for negative weights) is represented by a blunt end marker, i.e., a line segment perpendicular to the edge. The thickness of the edge also varies based on the magnitude of the absolute value of the weight. Larger magnitudes have thicker edges and smaller magnitudes have thinner edges. The way that GRNsight determines the edge thickness is as follows: GRNsight divides all weight values by the absolute value of the maximum weight in the adjacency matrix to normalize all the values to between zero and 1. GRNsight then adjusts the thickness of the lines to vary continuously from the minimum thickness (for normalized weights near zero) to maximum thickness (normalized weight of 1). The color of the edge also imparts information about the regulatory relationship. Edges with positive normalized weight values from 0.05 to 1 are colored magenta; edges with negative normalized weight values from -0.05 to -1 are colored cyan. Edges with normalized weight values between -0.05 and 0.05 are colored grey to emphasize that their normalized magnitude is near zero and that they have a weak influence on the target gene. When a user mouses over an edge, the numerical value of the weight parameter is displayed. When the the movements of the nodes. 
250

251

252

253

254

255

256

257

258

259

260

261

262

263

264

265

266

267

268

269

270

User Interface

The GRNsight user interface includes a menu/status bar and sliders that adjust D3.js's

force graph layout parameters. Figure 4 provides an annotated screenshot of the user interface, highlighting its primary features. Users can move force graph parameter sliders to refine the automated visualization. Nodes have a charge, which repels or attracts other nodes. The charge distance determines at what range a node's charge will affect other nodes. The link distance determines the minimum distance maintained between nodes. Gravity determines the strength of the force drawing the nodes to the center of the graph. Sliders can be locked to prevent changes and also reset to default values. Graph visualizations can also be modified through manual node dragging. Design decisions for the user interface were driven by applicable interaction design guidelines and principles (Nielsen, 1993; Shneiderman et al., 2016; Norman, 2013) in alignment with the mental model and expectations of the target user base, consisting primarily of biologists, both novice and experienced.

\section{Test-driven Development}

GRNsight follows an open development model with rigorous documentation of requirements and issues on GitHub. We have implemented an exhaustive unit testing framework using Mocha (https://mochajs.org) and the Chai assertion library (http://chaijs.com) to perform test-driven development where unit tests are written before new functionality is coded (Martin, 2008). This framework consists of around 160 automated unit tests that examine nearly 530 test files to ensure that the program is running as expected. Table 1 shows the test suite's coverage report, as generated by Istanbul (https://gotwarlost.github.io/istanbul/). 
Error and warning messages have a three-part framework that informs the user what

272 happened, the source of the problem, and possible solutions. This structure follows the alert

273 elements recommended by user interface guideline documents such as the OS X Human

274 Interface Guidelines

275 (https://developer.apple.com/library/mac/documentation/UserExperience/Conceptual/OSXHIGui

276 delines/WindowAlerts.html). For example, GRNsight returns an error when the spreadsheet is

277 formatted incorrectly or the maximum number of nodes or edges is exceeded.

278 Availability

279

GRNsight (currently version 1.18.1) is available at http://dondi.github.io/GRNsight/and is

280

281

282

283

284

285

286

287

288

289

290

291

compatible with Google Chrome version 43.0.2357.65 or higher and Mozilla Firefox version

38.0.1 or higher on the Windows 7 and Mac OS X operating systems. The website is free and open to all users, and there is no login requirement. Website content is available under the Creative Commons Attribution Non-Commercial Share Alike 3.0 Unported License. GRNsight code is available under the open source BSD license from our GitHub repository https://github.com/dondi/GRNsight. Every user's submitted data are private and not viewable by anyone other than the user. Uploaded data reside as temporary files and are deleted from the GRNsight server during standard operating system file cleanup procedures. A Google Analytics page view counter was implemented on 18 September 2014, and a file upload counter was added on 13 April 2015. From these start dates and as of 12 August 2016, the GRNsight home page has been accessed 2349 times, and 1652 files have been uploaded and viewed with GRNsight. Of these 1652 files, an estimated 148 were uploaded by users outside of our group. 
292 Results and Discussion

We have successfully implemented GRNsight, a web application and service for

visualizing small- to medium-scale gene regulatory networks, fulfilling our five requirements:

1. It exists as a web application without the need to download and install specialized software;

2. It is simple and intuitive to use;

3. It accepts an input file in Microsoft Excel format (.xlsx), as well as SIF (.sif) and GraphML (.graphml);

4. It reads a weighted or unweighted adjacency matrix where the regulatory transcription factors are in columns and the target genes are in rows (Excel format-only);

5. It automatically lays out and displays small- to medium-scale, unweighted and weighted, regulatory network graphs. Our discussion focuses on two of the demonstration files provided in the user interface, Demo \#3: Unweighted GRN (21 genes, 31 edges) and Demo \#4: Weighted GRN (21 genes, 31 edges, Schade et al. 2004 data). These two files describe gene regulatory networks from budding yeast, Saccharomyces cerevisiae, correspond to supplementary data

311 published by Dahlquist et al. (2015), and when displayed by GRNsight, represent interactive 312 versions of Figures 1 and 8 of that paper, respectively. 
Figure 5 gives a side-by-side view of the same adjacency matrices laid out by GRNsight and by hand. Figures 5A, 5B, and 5C are derived from Demo \#3: Unweighted GRN (21 genes, edges, Schade et al. 2004 data). Figures 5A and 5D show examples of the automatic layout performed by GRNsight. Figures 5C and 5F show the same adjacency matrices laid out by hand in Adobe Illustrator, corresponding to Figure 1 and Figure 8 of Dahlquist et al. (2015), respectively. Figures 5B and 5E started with the automatic layout from GRNsight and then were manually manipulated from within GRNsight to lay them out similarly to Figures 5C and 5F, respectively. The use of GRNsight represents a substantial time savings compared to creating the same figures entirely by hand and allows the user to try multiple arrangements of the nodes quickly and easily. Note that this type of "by hand" manipulation of graphs is most useful for small- to medium-scale networks, the kind that GRNsight is designed to display, and would not be appropriate for large networks. the network structure (Dahlquist et al., 2015). For example, YAP6 has the highest in-degree, being regulated by six other transcription factors. RAP1 has the highest out-degree of five, regulating four other transcription factors and itself. Four genes, AFT1, NRG1, RAP1, and YAP6, regulate themselves. Many of the transcription factors are involved in regulatory chains, with the longest including five nodes originating at SKN7 or ACE2. There are several other 4node chains that originate at CIN5, MAC1, PHD1, SKN7, and YAP1. Finally, there are two rather complex feedforward motifs involving CIN5, ROX1, and YAP6 and SKN7, YAP1, and ROX1 (Dahlquist et al., 2015). 
The networks with colored edges (Fig. 5D, E, F) display the results of a mathematical

336

337

338

339

340

341

342

343

344

345

346

347

348

349

350

351

352

353

354

355

356

357

model, where the expression levels of the individual transcription factors were modeled using

mass balance ordinary differential equations with a sigmoidal production function and linear degradation (Dahlquist et al., 2015). Each equation in the model included a production rate, a degradation rate, weights that denote the magnitude and type of influence of the connected transcription factors (activation or repression), and a threshold of expression. The differential equation model was fit to published yeast cold shock microarray data from Schade et al. (2004) using a penalized nonlinear least squares approach. The visualization produced by GRNsight is displaying the results of the optimized weight parameters. Positive weights $>0$ represent an activation relationship and are shown by pointed arrowheads. One example is that CIN5 activates the expression of MSN1. Negative weights $<0$ represent a repression relationship and are shown by a blunt arrowhead. One example is that ABF1 represses the expression of MSN1. The thicknesses of the edges also vary based on the magnitude of the absolute value of the weight, with larger magnitudes having thicker edges and smaller magnitudes having thinner edges. In Figures 5D, E, and F, the edge corresponding to the repression of the expression of MSN1 by ABF1 stands out as the thickest because the absolute value of its weight parameter (2.97) has the largest magnitude out of all the weights (Dahlquist et al., 2015). It is noticeable that none of the edges that represent activation are as thick as the ABF1-to-MSN1 edge; only RAP1-to-RPH1 and HAL9-to-MSN4 are close with weights of 1.50 and 1.43, respectively. with positive normalized weight values from 0.05 to 1 are colored magenta (10 edges in this example); edges with negative normalized weight values from -0.05 to -1 are colored cyan (16 edges in this example). Edges with normalized weight values between -0.05 and 0.05 are colored 
358

359

360

361

362

363

364

365

366

367

368

369

370

371

372

373

374

grey to indicate that their normalized magnitude is near zero and that they have a weak influence on the target gene ( 5 edges in this example). The grey color de-emphasizes the weak relationships to the eye, thus emphasizing the stronger colored relationships.

Because of this visualization of the weight parameters, one can make some interesting observations about the behavior of the network (Dahlquist et al., 2015). Taking the arrowhead type, thickness, and color into consideration, one can, by visual inspection, group edges by type and relative influence into four activation and four repression bins. RAP1-to-RPH1, HAL9-toMSN4, and NRG1 to itself have the strongest activation relationships, followed by CIN5-toMSN1, followed by NRG1-to-YAP6, MSN4-to-FHL1, SKN7-to ROX1 and PHD1-to-MSN4, followed by ABF1-to-FHL1 as the weakest of the activation relationships. The aforementioned ABF1-to-MSN1 edge has the strongest repression relationship, followed by ACE2-to-YAP1, RAP1-to-HSF1, CIN5-to-ROX1, AFT1 to itself, and RAP1 to itself, followed by ROX1-toYAP6, PHD1-to-CUP9, CIN5-to-YAP6, YAP6-to-ROX1, YAP1-to-ROX1, SKN7-to-YAP1, RAP1-to-AFT1, and YAP6 to itself, followed by MAC1-to-CUP9 and SKN7-to-NRG1 as the weakest of the repression relationships. These rankings could have been obtained, of course, by sorting the numerical values of the edges in a table, but it is notable that these groupings can also be picked out by eye and then put into the context of the other network connections.

Because the five weakest connections, CUP9-to-YAP6, REB1-to-GTS1, YAP6-to-CIN5, YAP1-to-YAP6, and HSF1-to-REB1, colored grey, are de-emphasized in the visual display, a different interpretation of the network structure can be made as compared to the unweighted network (Fig. 5E and F versus 5B and C). In most cases, nodes in a regulatory chain "drop out" visually "breaking" the chain. For example, in the four-node chain beginning with RAP1-toHSF1, the last two nodes, REB1 and GTS1, are only weakly connected. In the five-node chains 
381 beginning with SKN7-to-YAP1 or ACE2-to-YAP1, and the four-node chains beginning with

382 MAC1-to-CUP9 or PHD1-to-CUP9, the nodes connected to YAP6 drop out (YAP1-to-YAP6,

383 YAP6-to-CIN5, and CUP9-YAP6). This suggests that regulatory chains may only be effective

384 to a depth of two levels, and that while longer chains are theoretically possible, given the

385 network connections, they have a negligible effect on the dynamics of expression of downstream

386 genes.

Another interpretation of the network structure that is highlighted by the weighted display is that the 21-gene network can be divided into two smaller subnetworks by removing the two edges CUP9-to-YAP6 (grey) and ABF1-to-FHL1 (thin magenta, weakly activating). While this could also be observed in the unweighted network, the application of the weight information, showing only thin connections between the two subnetworks, suggests that they could function relatively independently. Finally, the unweighted display showed two complex feedforward motifs involving CIN5, ROX1, and YAP6 and SKN7, YAP1, and ROX1. The weighted display reveals that the complexity of the connections is reduced because the weak YAP1-to-YAP6 and YAP6-to-CIN5 edges drop out. Furthermore, the display shows that the modeling predicts that the three-node CIN5-ROX1-YAP6 motif is an incoherent type 2 feedforward loop, while the SKN7-YAP1-ROX1 motif is a coherent type 4 feedforward loop, neither of which is found very commonly in Escherichia coli nor S. cerevisiae gene regulatory networks (Alon, 2007). The modeling combined with the display suggests that further investigation is warranted: either these two rare types of feedforward loops are important to the dynamics of this particular GRN, or the

401 network structure is incorrect. In either case, future lines of experimental investigation are 402 suggested to the user. 
When examining individual genes in the network, one can see that the expression of

404

405

406

407

408

409

410

411

412

413

414

415

416

417

418

419

420

421

422

423

424

425

several genes is controlled by a balance of activation and repression by different regulators. For example, the expression of MSN1 is strongly activated by CIN5, but even more strongly repressed by ABF1. The expression of ROX1 is weakly activated by SKN7 and weakly repressed by YAP1, CIN5, and YAP6. The expression of YAP6 is weakly activated by NRG1, but weakly repressed by itself, CIN5, and ROX1. Furthermore, some transcription factors act both as activators of some targets and repressors of other targets. For example, RAP1 activates the expression of MSN4 and RPH1, but represses the expression of AFT1, HSF1, and itself. PHD1, ABF1, CIN5, and SKN7 also both activate and repress their different target genes in the network. For each of these regulators, there is experimental evidence to support their opposite effects on gene expression, although not necessarily for these particular target genes (RAP1: Shore and Nasmyth, 1987; PHD1: Borneman et al. 2006, ABF1: Buchman and Kornberg, 1990 and Miyake et al., 2004; CIN5 and SKN7: Ni et al., 2009). Except for CIN5, what these genes have in common is that they themselves have no inputs in the network. The remaining no-input genes (ACE2, MAC1, and HAL9) have only one outgoing edge in this network. Because these genes have no inputs and, in some sense, have been artificially disconnected from the larger GRN of the cell, one must not overinterpret the results of the modeling for these genes.

Thus, GRNsight enables one to interpret the weight parameters more easily than one could from the adjacency matrix alone. Visual inspection has long been recognized by experts such as Tufte (1983) and Card, Mackinlay, and Shneiderman (1999) as distinct from other forms of purely numeric, computational, or algorithmic data analysis, and as the preceding discussion highlights, it is this potential that can be derived specifically by visual inspection that is enabled by GRNsight. Card, Mackinlay, and Shneiderman (1999) have identified six major ways, 
426 documented in earlier literature and empirical studies, by which information visualization

427 amplifies cognition. Tufte's seminal book The Visual Display of Quantitative Information

428 (1983) perhaps states it best: "Graphics reveal data. Indeed graphics can be more precise and

429 revealing than conventional statistical computations."

Note that the nodes in Figure 5F are also colored in the style of GenMAPP 2 (Salomonis

431

432

433

434

435

436

437

438

439

440

441

442

443

444

445

446

447

448

et al., 2007), based on the time course of expression of that gene in the Schade et al. (2004)

microarray data (stripes from left to right, 10, 30, and 120 minutes of cold shock, with magenta representing a significant increase in expression relative to the control at time 0 , cyan

representing a significant decrease in expression relative to the control, and grey representing no significant change in expression relative to the control). This feature has not yet been implemented in GRNsight, but is currently under development for Version 2.

These observations made by direct inspection of the GRNsight graph are for a relatively small GRN of 21 genes and 31 edges and become more difficult as nodes and edges are added. For much larger networks, a more powerful graph analysis tool such as Cytoscape (Shannon et al., 2003; Smoot et al., 2011) or Gephi (Bastian, Heymann, and Jacomy, 2009) is warranted. However, for small networks in the range of 15-35 nodes, GRNsight fulfills a need to quickly and easily view and manipulate them. The GRN modeled in Dahlquist et al. (2015) and displayed in Figure 5 was derived by hand from the Lee et al. (2002) and Harbison et al. (2004) datasets generated by chromatin immunoprecipitation followed by microarray analysis. We have also used GRNsight to display GRNs derived from the YEASTRACT database (Teixeira et al., 2014), whose own display tool is static, displaying regulators and targets in two rows.

Instructions for viewing YEASTRACT-derived GRNs can be found on the GRNsight documentation page. 
While GRNsight was designed originally for viewing gene regulatory networks, it is not

450

451

452

453

454

455

456

457

458

459

460

461

462

463

464

465

466

467

468

469

470

471

specific for any particular species, nor for that kind of data. As long as the text strings used as

identifiers for the "regulators" and "targets" match, it can be used to visualize any small,

unweighted or weighted network with directed edges for systems biology or other application domains.

GRNsight Development Follows Best Practices for Scientific Computing and FAIR Data Principles

Veretnik, Fink, and Bourne (2008) lament and Schultheiss et al. (2011) document that some computational biology resources, especially web servers, lack persistence and usability, leading to an inability to reproduce results. With that in mind, we have consciously followed best practices for open development (Prlic and Procter, 2012), scientific computing (Wilson et al., 2014), providing a web resource (Schultheiss, 2011), and FAIR data (Wilkinson et al., 2016), simultaneously following and teaching these practices to the primary developers who were all undergraduates. Each of these practices relates to each other, supporting reproducible research.

Open Development and Long-term Persistence

As noted in our process requirements in the Introduction, we have followed an open development model since the project's inception in January 2014, with our code available under the open source BSD license at the public GitHub repository, where we "release early, release often” (Torvalds in Raymond, 1999) and also track requirements, issues, and bugs. Indeed, our project stands on the shoulders of other open source tools. Our unit-testing framework provides confidence that the code works as expected. Detailed documentation for users (web page) and developers (wiki) are provided. Demo data are also provided so users have both an example of how to format input files and can see how the software should perform. As noted by Prlic and 
472 Procter (2012), open development practices have a positive impact on the long-term

473 sustainability of a project. Furthermore, Schultheiss et al. (2011) describe twelve qualities for

474 evaluating web services that sum to a Long-Term-Score, which correlates with persistence of the

475 web service. GRNsight complies with all twelve requirements, providing: a stable web address

476 (using the github.io domain to host the website and Amazon Cloud Services to host the server

477 help to ensure long-term availability), version information, hosting country and institution, last

478 updated date, contact information, high usability, no registration requirement, no download

479 required, example data, fair testing possibility (both with demonstration Excel workbooks and

480 standard SIF and GraphML file types), and a functional service.

We are committed to continue development of the GRNsight resource, fixing bugs and improving the software by adding features. The lead authors (Dahlquist, Dionisio, and Fitzpatrick) are all tenured faculty, overseeing the design, code, testing, and documentation of GRNsight and providing continuity to the project. Together we have mentored the undergraduates (Anguiano, Varshneya, Southwick, and Samdarshi) who had primary responsibility for coding, testing, and documentation, while also being full partners in the design of the software. A pipeline has been established for onboarding new members to the project, also providing continuity. Lawlor and Walsh (2015) detail some of the same issues of reliability and reproducibility in bioinformatics software referred to by Wilson et al. (2014). Lawlor and Walsh (2015) conclude that the ideal way to bring software engineering values into bioinformatics research projects is to establish separate specialists in bioinformatics engineering.

492 We disagree. Through GRNsight, we have shown how best practices can be taught to undergraduates concomitant with training in bioinformatics, as we have shown previously with

494 Master's level students (Dionisio and Dahlquist, 2008). 
495

496

497

498

499

500

501

502

503

504

505

506

507

508

509

510

511

512

513

514

515

516

517

\section{FAIR Data Principles}

The FAIR Guiding Principles for scientific data and stewardship state that data should be Findable, Accessible, Interoperable, and Reusable by both humans and machines (Wilkinson et al., 2016), with "data" loosely construed as any scholarly digital research object, including software. As scientific software that interacts with data, the FAIR principles can apply to both the GRNsight application and the network data it is used to visualize. Thus, we evaluate the GRNsight project in terms of its "FAIRness" below.

\section{Findable}

The Findable principle states that metadata and data should have a globally unique and persistent identifier, and that metadata and data should be registered or indexed in a searchable resource (Wilkinson et al., 2016). In terms of software, the identifier is the name and version. Because we utilize the GitHub release mechanism, GRNsight code is tagged with a version (currently v1.18.1) and each version is available from the release page (https://github.com/dondi/GRNsight/releases). We have registered GRNsight with well-known bioinformatics tools registries: the BioJS Repository (Yachdav et al., 2015; http://biojs.io/), the Elixir Tools and Data Services Registry (Ison et al., 2016; https://bio.tools/), Bioinformatics.org (http://www.bioinformatics.org/wiki/), and the Links Directory at Bioinformatics.ca (Brazas, Yamada, and Ouellette, 2010), https://bioinformatics.ca/links_directory/), as well as NPM (Node Package Manager, https://www.npmjs.com/). GRNsight has also been presented at scientific conferences, with slides and posters available via SlideShare

(http://www.slideshare.net/GRNsight) and with a recent talk and poster at the 2016

Bioinformatics Open Source Conference available via F1000 Research (Dahlquist et al., 2016a; 2016b). We have paid special attention to the metadata associated with our website to increase 
518 its Findability via Google search. And, of course, with the publication of this article, GRNsight

519 is Findable in literature databases. In the everyday sense of the word "findable," one could argue

520 that by being "yet another" network visualization tool in a crowded domain (recall 47 other tools

521 recorded by Pavlopoulos et al., 2015), GRNsight is contributing to a Findability problem for

522 users in the sense that it contributes more "hay" to the "needle in a haystack" problem of finding

523 the right tool for the job. However, we hope that by the actions we have taken and the specificity

524 of our requirements for GRNsight's functionality, publicly describing both what we mean it to be

525 and what we do not mean it to be, the benefits of adding GRNsight to the diverse pool of

526 network visualization software outweighs the detriments.

In addition, the Findable principle states that data should be described with rich metadata

528

529

530

531

532

533

534

535

536

537

538

539

540

and that metadata should include the identifier of the data it describes (Wilkinson et al., 2016).

Because GRNsight does not interact directly with a data repository, it is up to individual users to make sure that their data is FAIR compliant with the Findable principle. This is discussed further below with regard to Interoperability and Reusability.

Accessible

The Accessible principle states that metadata and data should be retrievable by their identifier using a standardized communication protocol, that the protocol is open, free, and universally implementable, that the protocol allows for authentication and authorization procedures, where necessary, and that metadata are accessible, even when the data are no longer available (Wilkinson et al., 2016). As noted before, GRNsight meets the first two criteria, because it is free and open to all users, and there is no login requirement. The source code is available under the open source BSD license and can be npm installed (given the caveat that the user must be able to support the GRNsight client-server setup). The longevity of GRNsight is 
541 partially tied to the longevity of the GitHub repository itself, although the authors maintain local

542 backups. Again, because GRNsight does not interact directly with a data repository, it is up to

543 individual users to make sure that their data is FAIR compliant with the Accessible principle.

544 Since GRNsight does not have any security procedures nor authentication requirements (e.g.,

545 password protection; user registration), it is not recommended that sensitive data be uploaded to

546 our GRNsight server. However, users who wish to visualize sensitive data could run a local

547 instance of the GRNsight client-server setup.

548

549

550

551

552

553

554

555

556

557

558

559

560

561

562

563

Interoperable

As software, GRNsight does not interact directly with other databases or software, as, for example, Cytoscape does with many pathway and molecular interaction databases or individual Cytoscape apps (formerly plugins; Saito et al., 2012), so it is not Interoperable in that sense. The GRNsight web application is designed to interact directly with a human user and is not set up to import or export data programmatically, as would be necessary to incorporate it into popular workflow environments like Galaxy (Afgan et al., 2016) or be hosted by a tool aggregator such as QUBES Hub (Quantitative Undergraduate Biology Education and Synthesis Hub, https://qubeshub.org/). However, GRNsight is Interoperable in the sense that via the user, it can receive and pass data from and to other programs. In this latter sense, this section could just as easily have been entitled, " $95 \%$ of bioinformatics is getting your data into the right file format." Indeed, one of the original motivations and requirements for GRNsight was to seamlessly read and display weighted GRNs that were output as Excel workbooks from the GRNmap MATLAB modeling package (Dahlquist et al., 2015, http://kdahlquist.github.io/GRNmap/). This specialized use case is augmented by GRNsights's ability to import and export data in the commonly used SIF 
564 (http://manual.cytoscape.org/en/latest/Supported_Network_File_Formats.html\#sif-format) and

565 GraphML (Brandes, et al. 2001, http://graphml.graphdrawing.org/) formats, facilitating

566 movement of data between GRNsight and other network visualization and analysis programs.

567 For instance, one can interact with the GRNsight server component directly, in order to upload

568 Excel workbooks and supported import formats for conversion into JSON then back into a

569 supported export format. Thus, we are in a position to comment on SIF and GraphML with

570 respect to the finer points of data Interoperability, including: metadata and data using a formal,

571 accessible, shared, and broadly applicable language for knowledge representation, metadata and

572 data using vocabularies that follow the FAIR principles, and metadata and data including

573 qualified references to other metadata and data (Wilkinson et al., 2016).

When we implemented import and export for the SIF and GraphML formats, we

encountered issues due to the variations accepted by these formats which required design decisions that may, in turn, restrict compatibility with other software that we did not test. For example, the SIF format as described in the documentation for Cytoscape v3.4.0 offers quite a few divergent options, including choice of delimiter (space vs. tab), denoting a pairwise list of interactions versus concatenating all the interactions to the same node on the same line, and the choice of relationship type (any string). It only requires node identifiers to be internally consistent to the file, without enforcing the use of IDs from a recognized biological database.

While GRNsight strives to read any SIF file, we restricted our export format to tab-delimited, pairwise interactions, and a single relationship type ("pd" for "protein $\rightarrow$ DNA") for unweighted networks. For weighted networks, GRNsight exports the weight value as the relationship type. intended to encode is the interaction between two nodes, which makes including the weight data 
587 as GRNsight does a kludge, and including metadata impossible. Moreover, there is no controlled

588 vocabulary for the relationship type, only a list of suggestions in the Cytoscape documentation,

589 from which we selected "pd". In practice, Cytoscape v3.4.0 defaults to "interacts with" as the

590 relationship type when exporting SIF files. As a simple text format, it does not satisfy the three

591 sub-principles of Interoperability (Wilkinson et al. 2016).

In contrast, GraphML, as a richer XML format, has the potential to satisfy the

Interoperability criteria. However, as with SIF, we encountered issues because a feature of the

format that is intended to facilitate flexibility has, in practice, turned out to degrade

Interoperability rather than enhance it. GraphML standardizes only the representation of nodes and edges and their directions; all other characteristics, such as names, weights, and other values, are left for others to specify through a key element, which is not subject to a controlled vocabulary. Although this flexibility is appreciated, it also serves as an enabler for divergence.

In particular, two issues arose with interpreting the node identifier and display label. First, because of the lack of a controlled vocabulary, these are defined differently by different programs. Second, in the GRNsight-native Excel format, transcription factors must be unique in the header columns and rows and serve both as a unique ID for that node and the node label. In two implementations of GraphML import/export that we tested with Cytoscape v3.4.0 and a commercial graph editor called yED (v3.16, https://www.yworks.com/products/yed), an internal node ID is assigned independently of the node label and is not editable by the user. This leads to a situation where the user could assign identical labels to two or more nodes with different IDs, raising an issue for correct display of the network in GRNsight where node ID and node label are synonymous. GRNsight accommodates display of node labels from Cytoscape- and yED- 
610 encounter. Finally, as with SIF, there is no enforcement of the use of IDs from a recognized

611 biological database, even though the potential exists to specify the ID source (at least as a 612 comment) in the XML.

613 The format of a GraphML export by GRNsight is described on the Documentation page

614 (http://dondi.github.io/GRNsight/documentation.html). In our testing, we have ensured that

615 GRNsight can read Cytoscape- and yED-exported GraphML and that GRNsight-exported

616 GraphML was accurately read by these two programs, but we cannot guarantee Interoperability

617 with other software. Any issues that arise will need to be addressed on a case-by-case basis

618 through bug reports at our GitHub repository.

Compliance with FAIR principles is facilitated by the BioSharing registry of standards

620 (McQuilton et al., 2016; https://biosharing.org). As of this writing, GraphML is present in the 621 registry, but as an unclaimed, automatically-generated entry. Other formats for sharing network 622 data are potentially more fully FAIR compliant. However, the addition of each new format, 623 while increasing the flexibility and power of the GRNsight software, would incur the cost of 624 additional complexity (http://boxesandarrows.com/complexity-and-user-experience/). This is a 625 corollary of "one thing well" and is, for example, one reason why the complex Cytoscape stand626 alone application did not fit our initial product requirements. As demonstrated by our tests with 627 Cytoscape- and yED-exported GraphML, the aphorism that " $95 \%$ of bioinformatics is getting 628 your data into the right file format" cannot entirely be avoided by developers or users.

Reusable

The FAIR principles state that metadata and data should be richly described with a

631 plurality of accurate and relevant attributes, released with a clear and accessible usage license, 
632 associated with a detailed provenance, and meet domain-relevant community standards. As

633 software, GRNsight is Reusable because the code is available on GitHub under the open source

634 BSD license. The advantage of having followed test-driven development is that a developer who

635 wishes to reuse the code has a test suite ready to guide development of new features. In terms of

636 data, the criteria for Reusability are closely linked to Interoperability. While the GraphML

637 format is capable of storing metadata, the limitations described above in terms of a lack of

638 controlled vocabulary causes it to fail the Reusability test as well. In terms of provenance,

639 GRNsight injects a comment into the GraphML recording what version of GRNsight exported

640 the data (as does yED v3.16, but not Cytoscape v3.4.0). We also note that the GRNmap Excel

641 workbook format with multiple worksheets has the potential to record both metadata and

642 provenance, although this feature is not implemented at this time.

643 In the end, even the examples given by Wilkinson et al. (2016) have varying levels of

644 adherence to the FAIR principles or "FAIRness", which, they argue, should be used as a guide to

645 the incremental improvement of resources. Although GRNsight has the limitations discussed

646 above, we have done as much as we can to achieve FAIRness at this time.

647 Conclusions

We have successfully implemented GRNsight, a web application and service for

649 visualizing small- to medium-scale gene regulatory networks that is simple and intuitive to use.

650 GRNsight accepts an input file in Microsoft Excel format (.xlsx), reading a weighted or

651 unweighted adjacency matrix where the regulators are in columns and the target genes are in

652 rows, and automatically lays out and displays unweighted and weighted network graphs in a way

653 that is familiar to biologists. GRNsight also has the capability of importing and exporting files in 
654 SIF and GraphML formats. Although GRNsight was originally developed for use with the

655 GRNmap modeling software, and has provided useful insight into the interpretation of the gene 656 regulatory network model described in Dahlquist et al. (2015), it has general applicability for 657 displaying any small, unweighted or weighted network with directed edges for systems biology 658 or other application domains. Thus, GRNsight inhabits a niche not satisfied by other software, 659 doing "one thing well”. GRNsight also serves as a model for how best practices for software 660 engineering support reproducible research and can be learned simultaneously with the 661 development of useful bioinformatics software.

\section{Acknowledgments}

We would like to thank Katrina Sherbina and B.J. Johnson for their input during the early 664 stages of GRNsight development. We would also like to thank Masao Kitamura for assistance 665 with setting up and administering the GRNsight server. We thank the 2015-2016 GRNmap 666 research team, Chukwuemeka E. Azinge, Juan S. Carrillo, Kristen M. Horstmann, Kayla C.

667 Jackson, K. Grace Johnson, Brandon J. Klein, Tessa A. Morris, Margaret J. O’Neil, Trixie Anne 668 M. Roque, and Natalie E. Williams, and the students enrolled in the Loyola Marymount 669 University Spring 2015 course Biology 398-04: Biomathematical Modeling/Mathematics 388670 01: Survey of Biomathematics for testing the software. Finally, we thank Manuel Corpas and an 671 anonymous reviewer for suggestions that have improved both the GRNsight code and this 672 manuscript.

674 Afgan E., Baker D., van den Beek M., Blankenberg D., Bouvier D., Čech M., Chilton J., 
675

676

677

678

679

680

681

682

683

684

685

686

687

688

689

690

691

692

693

694

695

696

697

Clements D., Coraor N., Eberhard C., Grüning B., Guerler A., Hillman-Jackson J., Von Kuster G., Rasche E., Soranzo N., Turaga N., Taylor J., Nekrutenko A., Goecks J. 2016. The Galaxy platform for accessible, reproducible and collaborative biomedical analyses: 2016 update. Nucleic Acids Research 44:W3-W10. DOI: 10.1093/nar/gkw343.

Alon U. 2007. An introduction to systems biology: design principles of biological circuits. Boca Raton, FL: Chapman \& Hall/CRC. ISBN: 1-58488-642-0

Bastian M., Heymann S., Jacomy M. 2009. Gephi: an open source software for exploring and manipulating networks. Third International AAAI Conference on Weblogs and Social Media 8:361-362.

Borneman AR., Leigh-Bell JA., Yu H., Bertone P., Gerstein M., Snyder M. 2006. Target hub proteins serve as master regulators of development in yeast. Genes \& Development 20:435-448. DOI: 10.1101/gad.1389306.

Bostock M., Ogievetsky V., Heer J. 2011. D³: Data-Driven Documents. IEEE transactions on visualization and computer graphics 17:2301-2309. DOI: 10.1109/TVCG.2011.185.

Brandes U., Eiglsperger M., Herman I., Himsolt M., Marshall, MS. 2001. GraphML progress report structural layer proposal. In Graph Drawing: 9th International Symposium, GD 2001 Vienna, Austria, September 23-26, 2001 Revised Papers (pp. 501-512). Springer Berlin Heidelberg DOI: 10.1007/3-540-45848-4_59

Brazas MD., Yamada JT., Ouellette BFF. 2010. Providing web servers and training in Bioinformatics: 2010 update on the Bioinformatics Links Directory. Nucleic Acids Research 38:W3-6. DOI: 10.1093/nar/gkq553.

Brown E. 2014. Web development with Node and Express. Beijing ; Sebastopol, CA: O’Reilly. ISBN: 978-1-4919-4930-6 
698 Buchman AR., Kornberg RD. 1990. A yeast ARS-binding protein activates transcription 699 synergistically in combination with other weak activating factors. Molecular and Cellular $700 \quad$ Biology 10:887-897.

701 Card SK., Mackinlay JD., Shneiderman B. 1999. Chapter 1: Information Visualization. In 702 Readings in Information Visualization: Using Vision to Think. San Diego, California:

703

704

705

706

707

708

709

710

711

712

713

714

715

716

717

718

719

720

Academic Press. ISBN: 978-1-5586-0533-6

Dahlquist KD., Fitzpatrick BG., Camacho ET., Entzminger SD., Wanner NC. 2015. Parameter Estimation for Gene Regulatory Networks from Microarray Data: Cold Shock Response in Saccharomyces cerevisiae. Bulletin of Mathematical Biology 77:1457-1492. DOI: 10.1007/s11538-015-0092-6.

Dahlquist KD., Fitzpatrick BG., Dionisio JDN. Anguiano NA., Carrillo JS., Roque TAM., Varshneya A., Samdarshi M., Azinge CE. 2016a. GRNmap and GRNsight: open source software for dynamical systems modeling and visualization of medium-scale gene regulatory networks [v1; not peer reviewed]. F1000Research 5(ISCB Comm J):1637 (slides) DOI: 10.7490/f1000research.1112534.1

Dahlquist KD., Fitzpatrick BG., Dionisio JDN., Anguiano NA., Carrillo JS., Morris TA., Varshneya A., Williams NE., Johnson KG., Roque TAM., Horstmann KM., Samdarshi M., Azinge CE., Klein BJ., O'Neil MJ. 2016b. GRNmap and GRNsight: open source software for dynamical systems modeling and visualization of medium-scale gene regulatory networks [v1; not peer reviewed]. F1000Research 5(ISCB Comm J):1618 (poster) DOI: 10.7490/f1000research.1112518.1

Dionisio JDN., Dahlquist KD. 2008. Improving the computer science in bioinformatics through open source pedagogy. ACM SIGCSE Bulletin 40:115. DOI: 10.1145/1383602.1383648. 
721 Franz M., Lopes CT., Huck G., Dong Y., Sumer O., Bader GD. 2016. Cytoscape.js: a graph

722

723

724

725

726

727

728

729

730

731

732

733

734

735

736

737

738

739

740

741

742

743 theory library for visualisation and analysis. Bioinformatics (Oxford, England) 32:309311. DOI: 10.1093/bioinformatics/btv557.

Gostner R., Baldacci B., Morine MJ., Priami C. 2014. Graphical Modeling Tools for Systems Biology. ACM Computing Surveys 47:1-21. DOI: 10.1145/2633461.

Harbison CT., Gordon DB., Lee TI., Rinaldi NJ., Macisaac KD., Danford TW., Hannett NM., Tagne J-B., Reynolds DB., Yoo J., Jennings EG., Zeitlinger J., Pokholok DK., Kellis M., Rolfe PA., Takusagawa KT., Lander ES., Gifford DK., Fraenkel E., Young RA. 2004. Transcriptional regulatory code of a eukaryotic genome. Nature 431:99-104. DOI: 10.1038 /nature 02800 .

Ison J., Rapacki K., Ménager H., Kalaš M., Rydza E., Chmura P., Anthon C., Beard N., Berka K., Bolser D., Booth T., Bretaudeau A., Brezovsky J., Casadio R., Cesareni G., Coppens F., Cornell M., Cuccuru G., Davidsen K., Vedova GD., Dogan T., Doppelt-Azeroual O., Emery L., Gasteiger E., Gatter T., Goldberg T., Grosjean M., Grüning B., HelmerCitterich M., Ienasescu H., Ioannidis V., Jespersen MC., Jimenez R., Juty N., Juvan P., Koch M., Laibe C., Li J-W., Licata L., Mareuil F., Mičetić I., Friborg RM., Moretti S., Morris C., Möller S., Nenadic A., Peterson H., Profiti G., Rice P., Romano P., Roncaglia P., Saidi R., Schafferhans A., Schwämmle V., Smith C., Sperotto MM., Stockinger H., Vařeková RS., Tosatto SCE., de la Torre V., Uva P., Via A., Yachdav G., Zambelli F., Vriend G., Rost B., Parkinson H., Løngreen P., Brunak S. 2016. Tools and data services registry: a community effort to document bioinformatics resources. Nucleic Acids Research 44:D38-47. DOI: 10.1093/nar/gkv1116.

Lawlor B., Walsh P. 2015. Engineering bioinformatics: building reliability, performance and 
productivity into bioinformatics software. Bioengineered 6:193-203. DOI: 10.1080/21655979.2015.1050162.

746

747

748

749

750

751

752

753

754

755

756

757

758

759

760

761

762

763

764

765

766

Lee TI., Rinaldi NJ., Robert F., Odom DT., Bar-Joseph Z., Gerber GK., Hannett NM., Harbison CT., Thompson CM., Simon I., Zeitlinger J., Jennings EG., Murray HL., Gordon DB., Ren B., Wyrick JJ., Tagne J-B., Volkert TL., Fraenkel E., Gifford DK., Young RA. 2002. Transcriptional regulatory networks in Saccharomyces cerevisiae. Science (New York, N.Y.) 298:799-804. DOI: 10.1126/science.1075090.

Martin RC. (ed.) 2008. Clean code: a handbook of agile software craftsmanship. Upper Saddle River, NJ: Prentice Hall. ISBN: 978-0-13-235088-4

McQuilton P., Gonzalez-Beltran A., Rocca-Serra P., Thurston M., Lister A., Maguire E., Sansone S-A. 2016. BioSharing: curated and crowd-sourced metadata standards, databases and data policies in the life sciences. Database: The Journal of Biological Databases and Curation 2016. DOI: 10.1093/database/baw075.

Miyake T., Reese J., Loch CM., Auble DT., Li R. 2004. Genome-wide analysis of ARS (autonomously replicating sequence) binding factor 1 (Abflp)-mediated transcriptional regulation in Saccharomyces cerevisiae. The Journal of Biological Chemistry 279:3486534872. DOI: $10.1074 / \mathrm{jbc.M405156200.}$

Ni L., Bruce C., Hart C., Leigh-Bell J., Gelperin D., Umansky L., Gerstein MB., Snyder M. 2009. Dynamic and complex transcription factor binding during an inducible response in yeast. Genes \& Development 23:1351-1363. DOI: 10.1101/gad.1781909.

Nielsen J. 1993. Usability engineering. Boston: Academic Press. ISBN: 978-0-12-518405-2

Norman DA. 2013. The design of everyday things. New York, New York: Basic Books. ISBN: 978-0-465-05065-9 
767 Pavlopoulos GA., Malliarakis D., Papanikolaou N., Theodosiou T., Enright AJ., Iliopoulos I.

768 2015. Visualizing genome and systems biology: technologies, tools, implementation

769

770

771

772

773

774

775

776

777

778

779

780

781

782

783

784

785

786

787

788

789 techniques and trends, past, present and future. GigaScience 4:38. DOI: 10.1186/s13742015-0077-2.

Prlić A., Procter JB. 2012. Ten simple rules for the open development of scientific software. PLoS computational biology 8:e1002802. DOI: 10.1371/journal.pcbi.1002802.

Raymond ES. 1999. The cathedral \& the bazaar: musings on Linux and open source by an accidental revolutionary. Beijing ; Cambridge, Mass: O’Reilly. ISBN: 978-0-465-050659

Saito R., Smoot ME., Ono K., Ruscheinski J., Wang P-L., Lotia S., Pico AR., Bader GD., Ideker T. 2012. A travel guide to Cytoscape plugins. Nature Methods 9:1069-1076. DOI: 10.1038/nmeth.2212.

Salomonis N., Hanspers K., Zambon AC., Vranizan K., Lawlor SC., Dahlquist KD., Doniger SW., Stuart J., Conklin BR., Pico AR. 2007. GenMAPP 2: new features and resources for pathway analysis. BMC bioinformatics 8:217. DOI: 10.1186/1471-2105-8-217.

Schade B., Jansen G., Whiteway M., Entian KD., Thomas DY. 2004. Cold adaptation in budding yeast. Molecular Biology of the Cell 15:5492-5502. DOI: 10.1091/mbc.E04-03-0167.

Schultheiss SJ., Münch M-C., Andreeva GD., Rätsch G. 2011. Persistence and availability of Web services in computational biology. PloS One 6:e24914. DOI: 10.1371/journal.pone.0024914.

Schultheiss SJ. 2011. Ten simple rules for providing a scientific Web resource. PLoS computational biology 7:e1001126. DOI: 10.1371/journal.pcbi.1001126.

Shannon P., Markiel A., Ozier O., Baliga NS., Wang JT., Ramage D., Amin N., Schwikowski B., 
790

791

792

793

794

795

796

797

798

799

800

801

802

803

804

805

806

807

808

809

810

811

812

Ideker T. 2003. Cytoscape: a software environment for integrated models of biomolecular interaction networks. Genome Research 13:2498-2504. DOI: 10.1101/gr.1239303.

Shneiderman B., Plaisant C., Cohen M., Jacobs SM., Elmqvist N., Diakopoulos N. 2016. Designing the user interface: strategies for effective human-computer interaction. Hoboken: Pearson. ISBN: 978-0-13-438038-4

Shore D., Nasmyth K. 1987. Purification and cloning of a DNA binding protein from yeast that binds to both silencer and activator elements. Cell 51:721-732.

Smoot ME., Ono K., Ruscheinski J., Wang P-L., Ideker T. 2011. Cytoscape 2.8: new features for data integration and network visualization. Bioinformatics (Oxford, England) 27:431432. DOI: 10.1093/bioinformatics/btq675.

Teixeira MC., Monteiro PT., Guerreiro JF., Gonçalves JP., Mira NP., dos Santos SC., Cabrito TR., Palma M., Costa C., Francisco AP., Madeira SC., Oliveira AL., Freitas AT., SáCorreia I. 2014. The YEASTRACT database: an upgraded information system for the analysis of gene and genomic transcription regulation in Saccharomyces cerevisiae. Nucleic Acids Research 42:D161-166. DOI: 10.1093/nar/gkt1015.

Tufte ER. 2001. The visual display of quantitative information. Cheshire, Conn: Graphics Press. ISBN: 978-0-9613921-4-7

Veretnik S., Fink JL., Bourne PE. 2008. Computational biology resources lack persistence and usability. PLoS computational biology 4:e1000136. DOI: 10.1371/journal.pcbi.1000136.

Wilkinson MD., Dumontier M., Aalbersberg IJJ., Appleton G., Axton M., Baak A., Blomberg N., Boiten J-W., da Silva Santos LB., Bourne PE., Bouwman J., Brookes AJ., Clark T., Crosas M., Dillo I., Dumon O., Edmunds S., Evelo CT., Finkers R., Gonzalez-Beltran A., Gray AJG., Groth P., Goble C., Grethe JS., Heringa J., 't Hoen PAC., Hooft R., Kuhn T., 

P., Roos M., van Schaik R., Sansone S-A., Schultes E., Sengstag T., Slater T., Strawn G., Swertz MA., Thompson M., van der Lei J., van Mulligen E., Velterop J., Waagmeester A., Wittenburg P., Wolstencroft K., Zhao J., Mons B. 2016. The FAIR Guiding Principles for scientific data management and stewardship. Scientific Data 3:160018. DOI: $10.1038 /$ sdata.2016.18.

819 Wilson G., Aruliah DA., Brown CT., Chue Hong NP., Davis M., Guy RT., Haddock SHD., Huff KD., Mitchell IM., Plumbley MD., Waugh B., White EP., Wilson P. 2014. Best practices for scientific computing. PLoS biology 12:e1001745. DOI: 10.1371/journal.pbio.1001745.

Yachdav G., Goldberg T., Wilzbach S., Dao D., Shih I., Choudhary S., Crouch S., Franz M., García A., García LJ., Grüning BA., Inupakutika D., Sillitoe I., Thanki AS., Vieira B., 
Figure 1

Screenshot of the expected format for an adjacency matrix for an unweighted network.

Regulators are named in the columns and target genes in the rows. A gene name at the top of the matrix will be considered the same as a gene name on the side if it contains the same text string, regardless of capitalization.

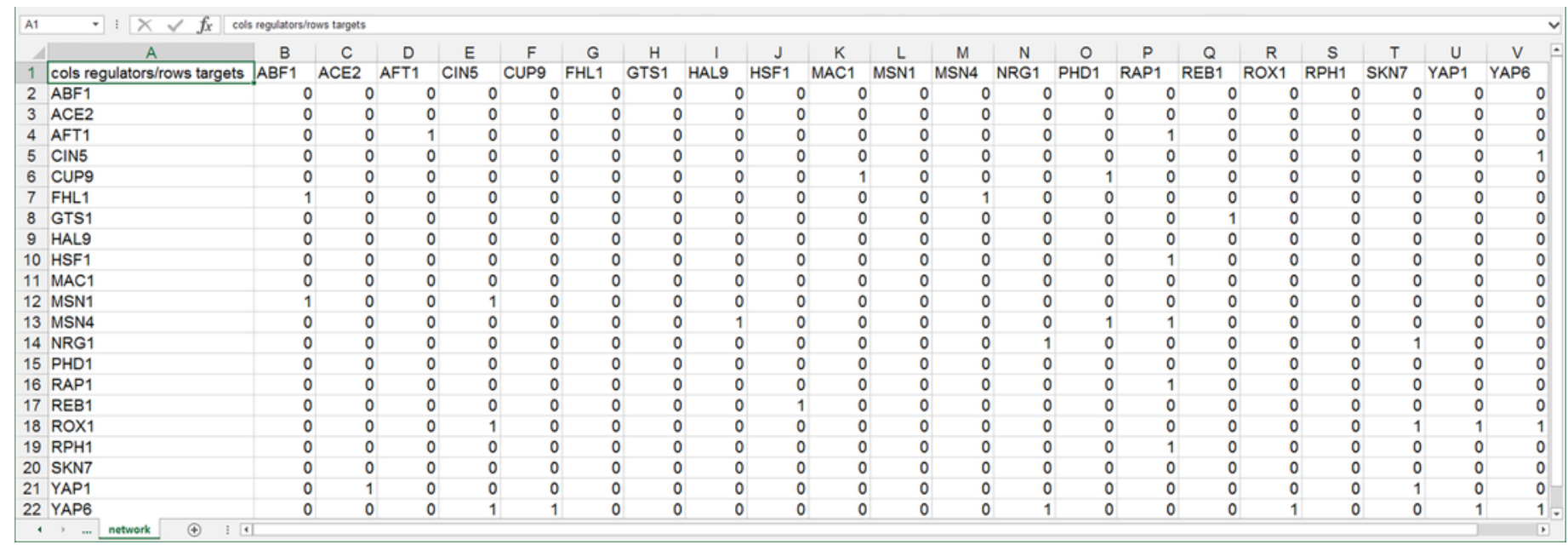


Figure 2

Screenshot of the expected format for an adjacency matrix for a weighted network.

Regulators are named in the columns and target genes in the rows. A gene name at the top of the matrix will be considered the same as a gene name on the side if it contains the same text string, regardless of capitalization.

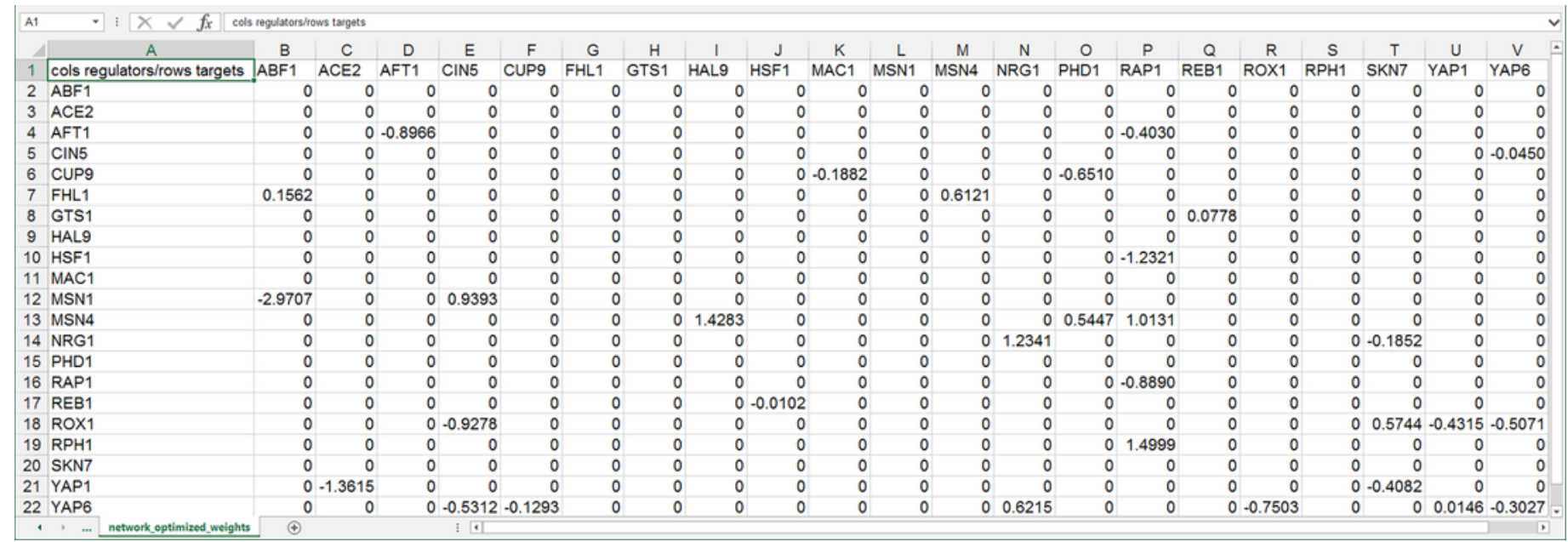


Figure 3 (on next page)

GRNsight architecture and component interactions. 


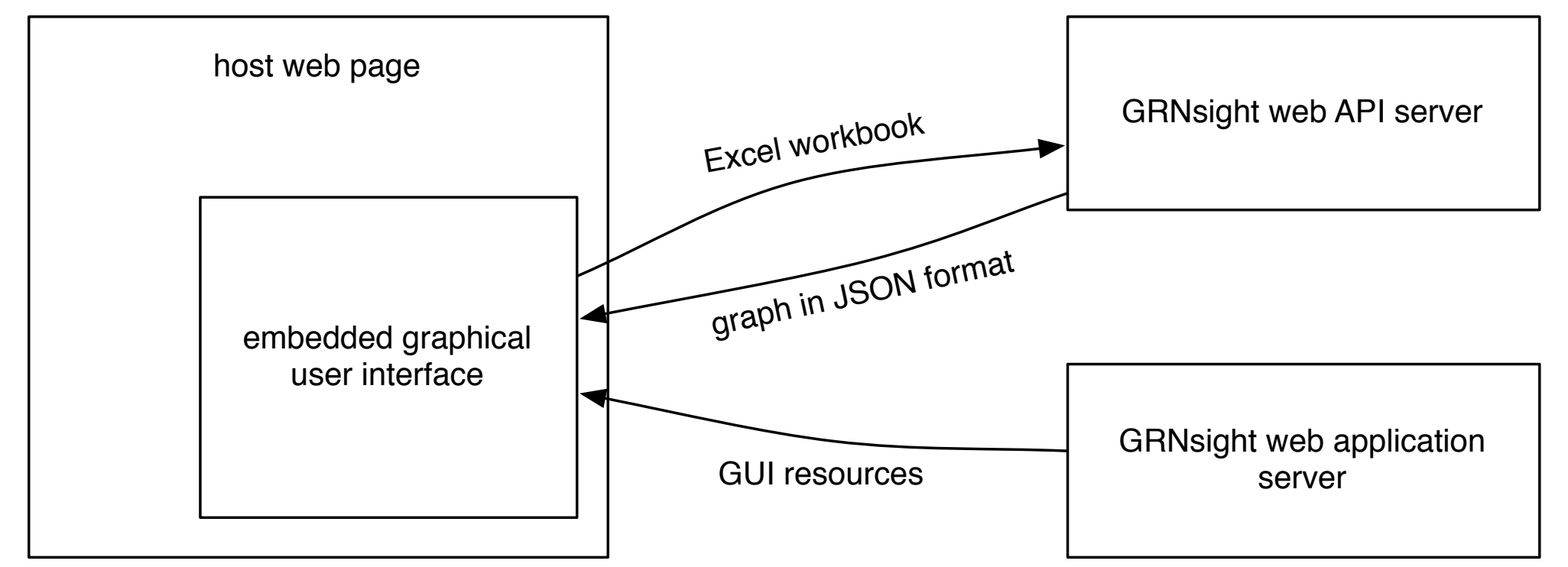


Figure 4 (on next page)

Annotated screenshot of the GRNsight user interface. 


\section{The File menu includes}

commands for uploading an adjacency matrix in Microsoft Excel (.xlsx) and other formats.

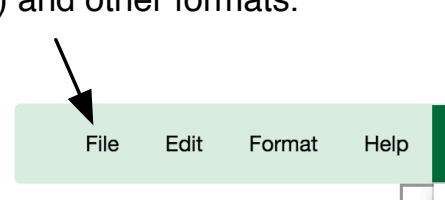

Link Distance (1 - 1000): 500

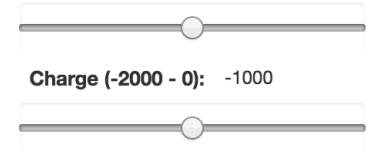

Charge Distance (0 - 2000): 1000

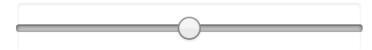

Gravity (0 - 1): 0.10

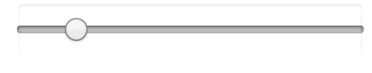

Lock Force Graph Parameters

Reset Force Graph Parameters

Undo Reset

Mouse over the edges to see the weight parameter values.

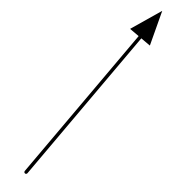

Force graph parameters can be adjusted, locked, or reset using this panel. Some commands are also available in the Format menu.

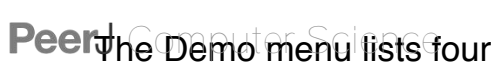
GRNs that have been preloaded into the server.
Manuscript to be reviewethe status display shows the current filename along with node and edge counts.

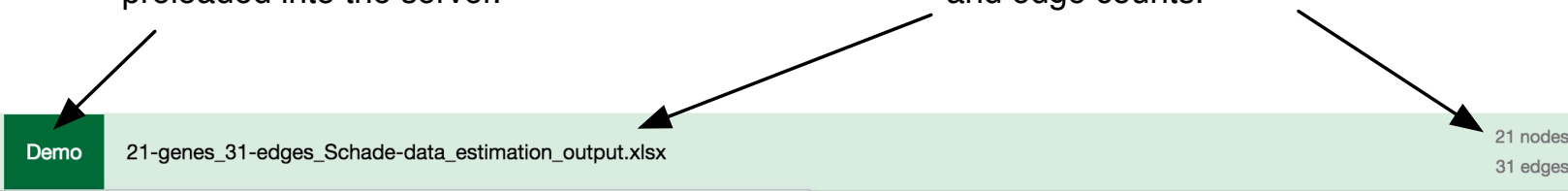

Demo \#1: Unweighted GRN (21 genes, 50 edges)

Demo \#2: Weighted GRN (21 genes, 50 edges, Dahlquist Lab unpublished data) Demo \#3: Unweighted GRN (21 genes, 31 edges)

Demo \#4: Weighted GRN (21 genes, 31 edges, Schade et al. 2004 data)

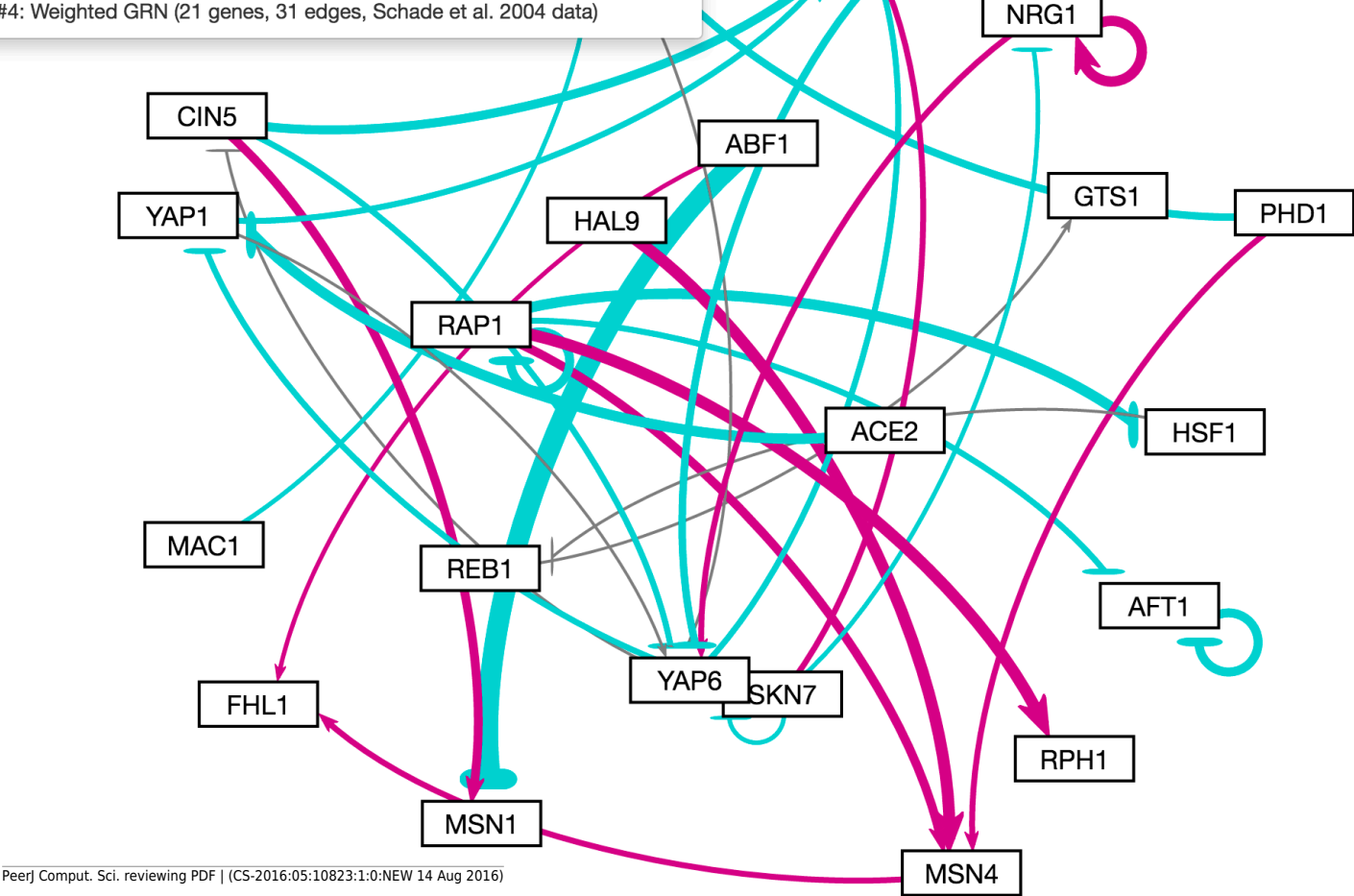




\section{Table $\mathbf{1}$ (on next page)}

GRNsight test suite code coverage summary.

Denominators represent the number of aspects of each type detected by Istanbul in the GRNsight codebase; numerators represent the subset of these which were executed by unit test code. 


\begin{tabular}{|l|l|}
\hline Aspect of the Code & Test Coverage (percent) \\
\hline Statements & $272 / 371(73.3 \%)$ \\
\hline Branches & $158 / 185(85.4 \%)$ \\
\hline Functions & $49 / 72(68.1 \%)$ \\
\hline Lines & $272 / 371(73.3 \%)$ \\
\hline
\end{tabular}

1 


\section{Figure 5 (on next page)}

Side-by-side comparison of the same adjacency matrices laid out by GRNsight and by hand.
A) GRNsight automatic layout of the demonstration file, Demo \#3: Unweighted GRN (21 genes, 31 edges); B) graph from (A) manually manipulated from within GRNsight; C) the same adjacency matrix from (A) and (B) laid out entirely by hand in Adobe Illustrator, corresponding to Figure 1 of Dahlquist et al., (2015); D) GRNsight automatic layout of the demonstration file, Demo \#4: Weighted GRN (21 genes, 31 edges, Schade et al. 2004 data); E) graph from (D) manually manipulated from within GRNsight; F) the same adjacency matrix from (D) and (E) laid out entirely by hand in Adobe lllustrator, corresponding to Figure 8 of Dahlquist et al., (2015). 


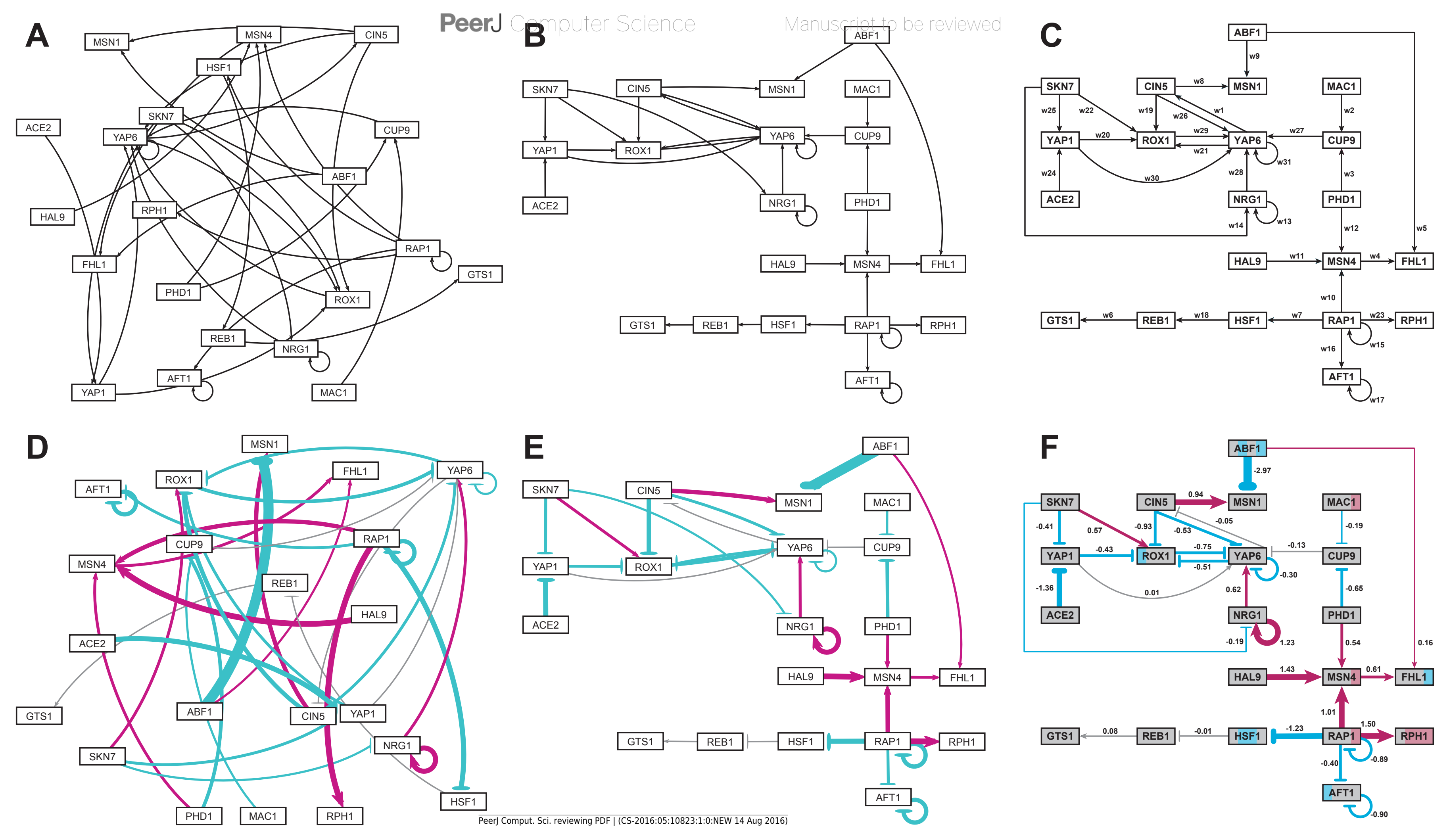

